\title{
Effect of Silver Nitrate and Silver Nanoparticles on Biofilm Formation in Staphylococcus Aureus
}

\author{
Muzafer Misic \\ Faculty of Engineering and Natural Sciences \\ Genetics and Bioengineering \\ International Burch University \\ Sarajevo, Bosnia and Herzegovina \\ Enisa Omanovic-Miklicanin \\ Faculty of Agriculture and Food Sciences \\ University of Sarajevo \\ Sarajevo, Bosnia and Herzegovina
}

\author{
Mirsada Hukic \\ Institute for Biomedical Diagnostics and Research NALAZ \\ Academy of Sciences and Arts \\ Sarajevo, Bosnia and Herzegovina \\ Monia Avdic \\ Faculty of Engineering and Natural Sciences \\ Genetics and bioengineering \\ International Burch University, \\ Sarajevo, Bosnia and Herzegovina
}

\begin{abstract}
Biofilms have become a major issue in different spheres of medicine and industry. Source of the issue revolves around increasing resistance of microorganisms towards conventional antibiotics which is even more elevated within biofilms that are difficult to eradicate by means of biocides and antibiotics. Multiple approaches were applied to deal with this issue amongst which green nanotechnology gave promising results and the search for molecules that "freeze bacteria" in the planktonic state. Since silver was used as an antimicrobial agent since ancient times. Considering this a study which deals with the effect of sub-inhibitory concentrations of silver nitrate and silver nanoparticles on biofilm forming capacity of bacteria would yield valuable information to evaluate the effect these substances have on the phenotypical expression of biofilm formation in bacteria as potential biofilm gene expression stoppers.
\end{abstract}

Keywords-: Silver, Silver nanoparticles, S. aureus, Green synthesis, Biofilm

\section{INTRODUCTION}

Biofilms are mono-species or multispecies microbial quorums, attached to a biotic or abiotic surface [1]. Within bacterial biofilms, bacteria have shown to express an altered phenotype compared to the planktonic state both in terms of morphology and gene expression [2]. An emphasized trait that is expressed in bacteria within the biofilm state is elevated resistance rates to antibiotics and biocides [3]. Considering this - biofilms today represent a greater challenge for eradication by different antimicrobial agents compared to planktonic bacteria [4].

Many agents were tested for the purpose of eliminating biofilms, i.e. antibiotics such as $\beta$-lactams, glycopeptides and disinfectants such as Benzalkonium chloride C12-18 (Campanac et al.2002); Chlorine (Grobe et al. 2002); Sodium hypochlorite (Bardouniotis et al. 2003). However, the search for novel substances for the eradication of biofilms is still ongoing.

Up to now the following metals were tested for their antibiofilm effect: silver, zinc, titanium, copper and gallium [5].

Due to toxicity and heavy metal poisoning metals such as gallium are not adequate for use, while the effect of other metals such as copper and zinc in the human body is not yet studied in detail [5].

Silver toxicity towards human cell line is much lower compared to bacteria. Considering this it is no surprise that silver was used as a sacred material in ancient times and as such earned its reputation as a ward against sickness [6].

Bacterial enzymes have become a target for various studies which aim to examine the interaction between silver and the enzymes. So far the main focus were the enzymes which possess the thiol groups [7]. It is presumed that the silver ions affect the enzyme itself by binding to the functional group [7]. Additionally silver ions cause release of $\mathrm{K}+$ ions which affects directly the cell wall and cell membrane of bacteria [7]. Silver has also shown to inhibit the growth of bacteria, damage the genetic material rendering the division useless and damage the cell wall forming abnormalities which in turn lead to loss of functionality [7].

The silver nanoparticles have proven antimicrobial effect on the various bacterial species tested. The mechanisms revolve around hindering the bacterial metabolism and/or damage of genetic material inside thus preventing replication and further division [8].

Nanoparticles have been proven to make "cracks" inside the bacterial cell wall. The degree of damage is regulated and proportional to the concentration and the size of nanoparticles used [9]. The methodologies for synthesis of nanoparticles are divided on conventional and non-conventional. Conventional methods involve trisodium citrate, sodium borohydrite as organic reducers. On the other hand non-conventional methods involve irradiation, laser ablation, metal evaporation for reduction. Contrary to these approaches is green synthesis of nanoparticles which use environmental friendly sugars, plant extracts, bacteria and fungi in order to create the nanoparticles and keep them stable [10].

Multiple chemical reduction methods have been proposed as the possible solutions to the increasing problems which biofilms and microbes residing inside cause, however due to their toxic nature these methodologies are becoming obsolete [11]. Unlike that the use of green synthesis gives promising results for the production of nanoparticles where the methodology is considered more safe and affordable [12] 
The aim of this work is to compare the anti-biofilm effects of the silver nanoparticles obtained by green synthesis and conventional chemical reduction.

\section{MATERIALS AND METHODS}

\section{A. Bacterial Strains}

The tested bacterial strains included: Staphylococcus aureus ATCC (25923), clinical strain of Methycillin sensitive Staphylococcus aureus (MSSA) and clinical strain of Methyciline resistant Staphylococcus aureus (MRSA). The cultures were stored in Tryptic soy broth (TSB) with 50\% glycerol on -80 degrees Celsius. Prior to use the bacterial strains were thawed and inoculated into TSB with $1 \%$ glucose as supplement, followed by overnight incubation.

\section{B. Silver nitrate and silver nanoparticles}

Solution of silver nitrate purchased from Sigma-Aldrich was prepared at concentration of $5 \mathrm{mM}$ in $100 \mathrm{~mL}$ of TSB. The solution was encased in protective foil to reduce photosensitive reactions and it was stored at 6 degrees Celsius inside a cooling unit.

1) Green synthesis of nanoparticles using Aloe Vera

Synthesis of nanoparticles was done with use of leaf extract from Aloe Vera plant. Healthy leaf was selected, rinsed thoroughly and cut up in small pieces. From that, 10 grams was weighted and moved in a beaker. Leaflets were placed in water previously heated to 80 degrees Celsius and were set to cook for 20 minutes. The mixture was transferred and centrifuged at $12.000 \mathrm{rpm}$ for 15 minutes at temperature of 4 degrees Celsius. Post centrifugation the supernatant was filtered through $0.45 \mu \mathrm{m}$ filter paper carefully without disturbing the pellet. The plant extract was taken and $10 \mathrm{~mL}$ is added drop-wise into $90 \mathrm{~mL}$ of silver nitrate at concentration of $1 \mathrm{mM}$. The mixture changed in color providing visual result that the reaction occurred, the mixture was transferred into tubes which were wrapped in protective aluminum foil and stored accordingly.

2) Synthesis of silver nanoparticles using the method of chemical reduction

Conventional method for synthesis of silver nanoparticles used chemical reduction with sodium borohydride with assistance of stabilizing agents. A solution of $1 \mathrm{mM}$ silver nitrate in $100 \mathrm{~mL}$ distilled water was prepared, afterwards solution of $2 \mathrm{mM}$ sodium borohydride was made in $100 \mathrm{~mL}$ of distilled water. With use of burette $30 \mathrm{~mL}$ was removed in a separate beaker, inside the beaker was then added $10 \mathrm{~mL}$ of silver nitrate drop-wise approximately one drop per second. Sodium chloride served as a stabilizing halide which prevented particle agglomeration. The tubes were marked and stored in cold storage at 4 degrees Celsius.

3) MIC detection of and silver nanoparticles synthesized using chemical reduction and green synthesis

MIC was determined using broth microdilution method. Upon inoculation the plates were incubated for 20 hours. Post incubation plates were inoculated onto Mueller-Hinton agar plates which were then incubated at 37 degrees Celsius. The MIC was selected as the highest concentration where no growth was present. The first dilution $(0.625 \mathrm{mM})$ where growth was present was monitored and selected as subinhibitory concentration. The sub-inhibitory concentration for silver nanoparticles synthesized with two methods them being chemical reduction and green synthesis method and was selected as $1 \mathrm{mM}$ according to the literature sources.

\section{4) TCP method of biofilm quantification}

Effect of sub-inhibitory concentrations of $0.625 \mathrm{mM}$ (silver nitrate) and $1 \mathrm{mM}$ (silver nanoparticles) on biofilm formation in the tested bacterial strains was evaluated using a modification of the TCP method. The bacterial strains were thawed and inoculated into fresh TSB supplemented with $1 \%$ glucose and were incubated for 20 hours at the temperature of 37 degrees Celsius. The microtiter plates were divided in two series of quadruplets per column in which the upper quadruplet was inoculated with the adequate density of the tested bacterial strain while the bottom quadruplet served as the negative control.

Upon filling the microtiter plates and diluting the concentration of silver nitrate / silver nanoparticles, bacteria were added at the density of $0.5 \mathrm{McF}$ arland standard. In total there were 18 microtiter plates ( 3 per condition, 9 per day), each of the plates were inoculated on sterile Mueller-Hinton agar plates prior to washing and staining with $0.1 \%$ crystal violet.

Stained microtiter plates were then analyzed on ELISA plate reader at $635 \mathrm{~nm}$ to obtain the absorbance values which in turn indicated the intensity of formed biofilm. Biofilm forming capacity was categorized as non-adherent, weak, moderate, strong according to formulas in (Table 1)

\section{RESULTS}

\section{A. Effects silver nitrate and silver nanoparticles on biofilm forming capacity tested strains}

In positive control S. aureus (MSSA) did not form adherent biofilm in positive control, when exposed to silver nitrate at concentrations $0.3125 \mathrm{mM}, 0.001220 \mathrm{mM}$ and $0.00061 \mathrm{mM}$ the biofilm intensity changed from non-adherent to weak, posr 48 hour incubation the biofilm intensity changed from weak at mentioned concentrations to non-adherent. S. aureus (MRSA) did not form adherent biofilm in the positive control, exposure to silver nitrate did not change the intensity in any of the tested concentrations, post 48 hour incubation the outcome remained the same. S. aureus (ATCC 25923) formed biofilm of medium intensity inside the positive control, when exposed to the silver nitrate the biofilm was quenched at concentration of $0.625 \mathrm{mM}$ and the intensity was reduced to weak in other tested concentrations with exception at the concentration of $0.00975 \mathrm{mM}$ where the intensity was restored to medium, post 48 hours of exposure biofilm of weak intensity formed at concentrations of $0.625 \mathrm{mM}, 0.0195 \mathrm{mM}$, $0.00975 \mathrm{mM}, 0.00061 \mathrm{mM}$ (Table 2). 
Table 2- Effects of different sub-inhibitory concentrations of silver nitrate $(0.625 \mathrm{mM})$ on S. aurues (MSSA, MRSA, ATCC) after 24 hours and 48 hours

\begin{tabular}{|c|c|c|c|c|c|c|}
\hline \multirow{3}{*}{ 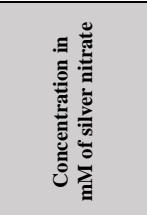 } & \multicolumn{2}{|c|}{$\begin{array}{l}\text { Staphylococcus } \\
\text { aureus (MSSA) }\end{array}$} & \multicolumn{2}{|c|}{$\begin{array}{l}\text { Staphylococcus } \\
\text { aureus (MRSA) }\end{array}$} & \multicolumn{2}{|c|}{$\begin{array}{c}\text { Staphylococcus } \\
\text { aureus (ATCC } \\
\text { 25923) }\end{array}$} \\
\hline & \multicolumn{6}{|c|}{ Biofilm forming capacity } \\
\hline & 24 hours & 48 hours & 24 hours & 48 hours & 24 hours & 48 hours \\
\hline (-) Control* & $\begin{array}{c}\text { Non- } \\
\text { adherent }\end{array}$ & $\begin{array}{c}\text { Non- } \\
\text { adherent }\end{array}$ & $\begin{array}{c}\text { Non- } \\
\text { adherent }\end{array}$ & $\begin{array}{c}\text { Non- } \\
\text { adherent }\end{array}$ & $\begin{array}{c}\text { Non- } \\
\text { adherent }\end{array}$ & $\begin{array}{c}\text { Non- } \\
\text { adherent }\end{array}$ \\
\hline (+) Control* & $\begin{array}{c}\text { Non- } \\
\text { adherent }\end{array}$ & $\begin{array}{c}\text { Non- } \\
\text { adherent }\end{array}$ & $\begin{array}{c}\text { Non- } \\
\text { adherent }\end{array}$ & $\begin{array}{c}\text { Non- } \\
\text { adherent }\end{array}$ & Medium & $\begin{array}{c}\text { Non- } \\
\text { adherent }\end{array}$ \\
\hline 0.625 & $\begin{array}{c}\text { Non- } \\
\text { adherent }\end{array}$ & $\begin{array}{c}\text { Non- } \\
\text { adherent }\end{array}$ & $\begin{array}{c}\text { Non- } \\
\text { adherent }\end{array}$ & $\begin{array}{c}\text { Non- } \\
\text { adherent }\end{array}$ & $\begin{array}{c}\text { Non- } \\
\text { adherent }\end{array}$ & Weak \\
\hline 0.3 & Weak & $\begin{array}{c}\text { Non- } \\
\text { adherent }\end{array}$ & $\begin{array}{c}\text { Non- } \\
\text { adherent }\end{array}$ & $\begin{array}{c}\text { Non- } \\
\text { adherent }\end{array}$ & Weak & $\begin{array}{c}\text { Non- } \\
\text { adherent }\end{array}$ \\
\hline 0.15625 & $\begin{array}{c}\text { Non- } \\
\text { adherent }\end{array}$ & $\begin{array}{c}\text { Non- } \\
\text { adherent }\end{array}$ & $\begin{array}{c}\text { Non- } \\
\text { adherent }\end{array}$ & $\begin{array}{c}\text { Non- } \\
\text { adherent }\end{array}$ & Weak & $\begin{array}{c}\text { Non- } \\
\text { adherent }\end{array}$ \\
\hline 0.0781 & $\begin{array}{c}\text { Non- } \\
\text { adherent }\end{array}$ & $\begin{array}{c}\text { Non- } \\
\text { adherent }\end{array}$ & $\begin{array}{c}\text { Non- } \\
\text { adherent }\end{array}$ & $\begin{array}{c}\text { Non- } \\
\text { adherent }\end{array}$ & Weak & $\begin{array}{c}\text { Non- } \\
\text { adherent }\end{array}$ \\
\hline 0.0390 & $\begin{array}{c}\text { Non- } \\
\text { adherent }\end{array}$ & $\begin{array}{c}\text { Non- } \\
\text { adherent }\end{array}$ & $\begin{array}{c}\text { Non- } \\
\text { adherent }\end{array}$ & $\begin{array}{c}\text { Non- } \\
\text { adherent }\end{array}$ & Weak & $\begin{array}{c}\text { Non- } \\
\text { adherent }\end{array}$ \\
\hline 0.0195 & $\begin{array}{c}\text { Non- } \\
\text { adherent }\end{array}$ & $\begin{array}{c}\text { Non- } \\
\text { adherent }\end{array}$ & $\begin{array}{c}\text { Non- } \\
\text { adherent }\end{array}$ & $\begin{array}{c}\text { Non- } \\
\text { adherent }\end{array}$ & Weak & Weak \\
\hline 0.00975 & $\begin{array}{c}\text { Non- } \\
\text { adherent }\end{array}$ & $\begin{array}{c}\text { Non- } \\
\text { adherent }\end{array}$ & $\begin{array}{c}\text { Non- } \\
\text { adherent }\end{array}$ & $\begin{array}{c}\text { Non- } \\
\text { adherent }\end{array}$ & Medium & Weak \\
\hline 0.0046875 & $\begin{array}{c}\text { Non- } \\
\text { adherent }\end{array}$ & $\begin{array}{c}\text { Non- } \\
\text { adherent }\end{array}$ & $\begin{array}{c}\text { Non- } \\
\text { adherent }\end{array}$ & $\begin{array}{c}\text { Non- } \\
\text { adherent }\end{array}$ & Weak & $\begin{array}{c}\text { Non- } \\
\text { adherent }\end{array}$ \\
\hline 0.002441 & $\begin{array}{c}\text { Non- } \\
\text { adherent }\end{array}$ & $\begin{array}{c}\text { Non- } \\
\text { adherent }\end{array}$ & $\begin{array}{c}\text { Non- } \\
\text { adherent }\end{array}$ & $\begin{array}{c}\text { Non- } \\
\text { adherent }\end{array}$ & Weak & $\begin{array}{c}\text { Non- } \\
\text { adherent }\end{array}$ \\
\hline 0.001220 & Weak & $\begin{array}{c}\text { Non- } \\
\text { adherent }\end{array}$ & $\begin{array}{c}\text { Non- } \\
\text { adherent }\end{array}$ & $\begin{array}{c}\text { Non- } \\
\text { adherent }\end{array}$ & Weak & $\begin{array}{c}\text { Non- } \\
\text { adherent }\end{array}$ \\
\hline 061 & Wc & $\begin{array}{c}\text { Non- } \\
\text { adherent }\end{array}$ & $\begin{array}{c}\text { Non- } \\
\text { adherent }\end{array}$ & $\begin{array}{c}\text { Non- } \\
\text { adherent }\end{array}$ & Weak & W \\
\hline
\end{tabular}

S. aureus (MSSA) in positive control did not form adherent biofilm, when exposed to silver nanoparticles synthesized using green synthesis method, at concentration $0.125 \mathrm{mM}$ the biofilm intensity changed from non-adherent to weak, post 48 hours the biofilm maintained its intensity at the same concentration. S. aureus (MRSA) in positive control did not form adherent biofilm, the intensity remained nonadherent through the tested concentrations, post 48 hour incubation the biofilm intensity changed from non-adherent to weak at the concentration of $0.5 \mathrm{mM}$. S. aureus (ATCC 25923) has formed inside the positive control biofilm of medium intensity, when exposed to silver nanoparticles at concentrations $1 \mathrm{mM}, 0.5 \mathrm{mM}$ and $0.25 \mathrm{mM}$ at which biofilm was quenched, the intensity was restored to medium at concentration $0.125 \mathrm{mM}$ and kept its intensity throughout the remaining concentrations, post 48 hour incubation the biofilm intensity inside the positive control changed from medium to non-adherent. This manifestation can be accredited to biofilm disintegration. Biofilm intensity changed at the concentrations of $0.25 \mathrm{mM}, 0.015625 \mathrm{mM}$ and $0.000977 \mathrm{mM}$ where the intensity changed from non-adherent to weak biofilm. (Table 3).
Table 3 - Effects of different sub-inhibitory concentrations of Aloe Vera synthesized nanoparticles (1 mM) on S. aurues (MSSA, MRSA, ATCC) after

\begin{tabular}{|c|c|c|c|c|c|c|}
\hline \multirow{3}{*}{ 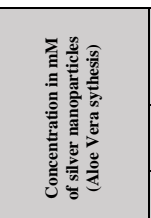 } & $\begin{array}{l}\text { Staph } \\
\text { aureu }\end{array}$ & $\begin{array}{l}\text { coccus } \\
\text { ISSA) }\end{array}$ & $\begin{array}{l}\text { Staph } \\
\text { aureu }\end{array}$ & $\begin{array}{l}\text { occus } \\
\text { IRSA) }\end{array}$ & \multicolumn{2}{|c|}{$\begin{array}{c}\text { Staphylococcus } \\
\text { aureus (ATCC } \\
\mathbf{2 5 9 2 3 )}\end{array}$} \\
\hline & \multicolumn{6}{|c|}{ Biofilm forming capacity } \\
\hline & 24 hours & 48 hours & 24 hours & 48 hours & 24 hours & 48 hours \\
\hline (-) Control* & $\begin{array}{c}\text { Non- } \\
\text { adherent }\end{array}$ & $\begin{array}{c}\text { Non- } \\
\text { adherent }\end{array}$ & $\begin{array}{c}\text { Non- } \\
\text { adherent }\end{array}$ & $\begin{array}{c}\text { Non- } \\
\text { adherent }\end{array}$ & $\begin{array}{c}\text { Non- } \\
\text { adherent }\end{array}$ & $\begin{array}{c}\text { Non- } \\
\text { adherent }\end{array}$ \\
\hline (+) Control* & $\begin{array}{c}\text { Non- } \\
\text { adherent }\end{array}$ & $\begin{array}{c}\text { Non- } \\
\text { adherent }\end{array}$ & $\begin{array}{c}\text { Non- } \\
\text { adherent }\end{array}$ & $\begin{array}{c}\text { Non- } \\
\text { adherent }\end{array}$ & Medium & $\begin{array}{c}\text { Non- } \\
\text { adherent }\end{array}$ \\
\hline 1 & $\begin{array}{c}\text { Non- } \\
\text { adherent }\end{array}$ & $\begin{array}{c}\text { Non- } \\
\text { adherent }\end{array}$ & $\begin{array}{c}\text { Non- } \\
\text { adherent }\end{array}$ & $\begin{array}{c}\text { Non- } \\
\text { adherent }\end{array}$ & $\begin{array}{c}\text { Non- } \\
\text { adherent }\end{array}$ & $\begin{array}{c}\text { Non- } \\
\text { adherent }\end{array}$ \\
\hline 0.5 & $\begin{array}{c}\text { Non- } \\
\text { adherent }\end{array}$ & $\begin{array}{c}\text { Non- } \\
\text { adherent }\end{array}$ & $\begin{array}{c}\text { Non- } \\
\text { adherent }\end{array}$ & Weak & $\begin{array}{c}\text { Non- } \\
\text { adherent }\end{array}$ & $\begin{array}{c}\text { Non- } \\
\text { adherent }\end{array}$ \\
\hline 0.25 & $\begin{array}{c}\text { Non- } \\
\text { adherent }\end{array}$ & $\begin{array}{c}\text { Non- } \\
\text { adherent }\end{array}$ & $\begin{array}{c}\text { Non- } \\
\text { adherent }\end{array}$ & $\begin{array}{c}\text { Non- } \\
\text { adherent }\end{array}$ & $\begin{array}{c}\text { Non- } \\
\text { adherent }\end{array}$ & Weak \\
\hline 0.125 & Weak & Weak & $\begin{array}{c}\text { Non- } \\
\text { adherent }\end{array}$ & $\begin{array}{c}\text { Non- } \\
\text { adherent }\end{array}$ & Medium & $\begin{array}{c}\text { Non- } \\
\text { adherent }\end{array}$ \\
\hline 0.0625 & \begin{tabular}{|c|} 
Non- \\
adherent
\end{tabular} & $\begin{array}{c}\text { Non- } \\
\text { adherent }\end{array}$ & $\begin{array}{c}\text { Non- } \\
\text { adherent }\end{array}$ & $\begin{array}{c}\text { Non- } \\
\text { adherent }\end{array}$ & Medium & $\begin{array}{c}\text { Non- } \\
\text { adherent }\end{array}$ \\
\hline 0.03125 & \begin{tabular}{|c|} 
Non- \\
adherent
\end{tabular} & $\begin{array}{c}\text { Non- } \\
\text { adherent }\end{array}$ & $\begin{array}{c}\text { Non- } \\
\text { adherent }\end{array}$ & $\begin{array}{c}\text { Non- } \\
\text { adherent }\end{array}$ & Medium & $\begin{array}{c}\text { Non- } \\
\text { adherent }\end{array}$ \\
\hline 0.015625 & $\begin{array}{c}\text { Non- } \\
\text { adherent }\end{array}$ & $\begin{array}{c}\text { Non- } \\
\text { adherent }\end{array}$ & $\begin{array}{c}\text { Non- } \\
\text { adherent }\end{array}$ & $\begin{array}{c}\text { Non- } \\
\text { adherent }\end{array}$ & Medium & Weak \\
\hline 0.007813 & $\begin{array}{c}\text { Non- } \\
\text { adherent }\end{array}$ & $\begin{array}{c}\text { Non- } \\
\text { adherent }\end{array}$ & $\begin{array}{c}\text { Non- } \\
\text { adherent }\end{array}$ & $\begin{array}{c}\text { Non- } \\
\text { adherent }\end{array}$ & Medium & $\begin{array}{c}\text { Non- } \\
\text { adherent }\end{array}$ \\
\hline 0.003906 & $\begin{array}{c}\text { Non- } \\
\text { adherent }\end{array}$ & $\begin{array}{c}\text { Non- } \\
\text { adherent }\end{array}$ & $\begin{array}{c}\text { Non- } \\
\text { adherent }\end{array}$ & $\begin{array}{c}\text { Non- } \\
\text { adherent }\end{array}$ & Medium & $\begin{array}{c}\text { Non- } \\
\text { adherent }\end{array}$ \\
\hline 0.001953 & \begin{tabular}{|c|} 
Non- \\
adherent
\end{tabular} & $\begin{array}{c}\text { Non- } \\
\text { adherent }\end{array}$ & $\begin{array}{c}\text { Non- } \\
\text { adherent }\end{array}$ & $\begin{array}{c}\text { Non- } \\
\text { adherent }\end{array}$ & Medium & $\begin{array}{c}\text { Non- } \\
\text { adherent }\end{array}$ \\
\hline 0.000977 & $\begin{array}{c}\text { Non- } \\
\text { adherent }\end{array}$ & $\begin{array}{c}\text { Non- } \\
\text { adherent }\end{array}$ & $\begin{array}{c}\text { Non- } \\
\text { adherent }\end{array}$ & $\begin{array}{c}\text { Non- } \\
\text { adherent }\end{array}$ & Medium & Weak \\
\hline
\end{tabular}

S. aureus MSSA and MRSA in positive controls did not form adherent biofilm, the intensity did not change in any of the tested concentrations of silver nanoparticles, after 48 hour incubation change of biofilm intensity occurred in $\mathrm{S}$. aureus (MRSA) in positive control where weak biofilm formed from non-adherent, other tested concentrations remained unchanged. S. aureus (ATCC 25923) in positive control formed medium intensity biofilm, change occurred at concentrations of $0.00195312 \mathrm{mM}$ and $0.00097656 \mathrm{mM}$ at which the intensity was changed to weak. Higher concentrations have quenched the biofilm thus no biofilm was present, post 48 hours incubation change in biofilm intensity occurred at positive control where biofilm has disintegrated, notable changes occurred at concentrations of $0.003906 \mathrm{mM}$ and $0.001953 \mathrm{mM}$ where the intensity changed from nonadherent to weak and medium intensity. Other concentration yielded no change in biofilm intensity from non-adherent (Table 4). 
Table 4 - Effects of different sub-inhibitory concentrations of nanoparticles synthesized by method of chemical reduction $(1 \mathrm{mM})$ on $\mathrm{S}$. aureus (MSSA MRSA, ATCC) after 24 hours and 48 hours

\begin{tabular}{|c|c|c|c|c|c|c|}
\hline \multirow{3}{*}{ 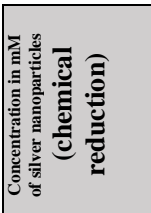 } & \multicolumn{2}{|c|}{$\begin{array}{l}\text { Staphylococcus } \\
\text { aureus (MSSA) }\end{array}$} & \multicolumn{2}{|c|}{$\begin{array}{l}\text { Staphylococcus } \\
\text { aureus (MRSA) }\end{array}$} & \multicolumn{2}{|c|}{$\begin{array}{c}\text { Staphylococcus } \\
\text { aureus (ATCC } \\
\mathbf{2 5 9 2 3 )}\end{array}$} \\
\hline & \multicolumn{6}{|c|}{ Biofilm forming capacity } \\
\hline & 24 hours & 48 hours & 24 hours & 48 hours & 24 hours & 48 hours \\
\hline (-) Control* & 0 & $\begin{array}{c}\text { Non } \\
\text { adherent }\end{array}$ & $\begin{array}{c}\text { Non- } \\
\text { adherent }\end{array}$ & $\begin{array}{c}\text { Non- } \\
\text { adherent }\end{array}$ & $\begin{array}{c}\text { Non- } \\
\text { adherent }\end{array}$ & $\begin{array}{c}\text { Non- } \\
\text { adherent }\end{array}$ \\
\hline (+) Control* & 0,0698 & $\begin{array}{c}\text { Non } \\
\text { adherent }\end{array}$ & $\begin{array}{c}\text { Non- } \\
\text { adherent }\end{array}$ & $\begin{array}{c}\text { Non- } \\
\text { adherent }\end{array}$ & Medium & $\begin{array}{c}\text { Non- } \\
\text { adherent }\end{array}$ \\
\hline 1 & 0,0745 & $\begin{array}{c}\text { Non } \\
\text { adherent }\end{array}$ & $\begin{array}{c}\text { Non- } \\
\text { adherent }\end{array}$ & $\begin{array}{c}\text { Non- } \\
\text { adherent }\end{array}$ & $\begin{array}{c}\text { Non- } \\
\text { adherent }\end{array}$ & $\begin{array}{c}\text { Non- } \\
\text { adherent }\end{array}$ \\
\hline 0.5 & 58 & $\begin{array}{c}\text { Non } \\
\text { adherent }\end{array}$ & $\begin{array}{c}\text { Non- } \\
\text { adherent }\end{array}$ & $\begin{array}{c}\text { Non- } \\
\text { adherent }\end{array}$ & $\begin{array}{c}\text { Non- } \\
\text { adherent }\end{array}$ & $\begin{array}{c}\text { Non- } \\
\text { adherent }\end{array}$ \\
\hline 0.25 & 560 & $\begin{array}{c}\text { Non } \\
\text { adherent }\end{array}$ & $\begin{array}{c}\text { Non- } \\
\text { adherent }\end{array}$ & $\begin{array}{c}\text { Non- } \\
\text { adherent }\end{array}$ & $\begin{array}{c}\text { Non- } \\
\text { adherent }\end{array}$ & $\begin{array}{c}\text { Non- } \\
\text { adherent }\end{array}$ \\
\hline 0.125 & 570 & $\begin{array}{c}\text { Non } \\
\text { adherent }\end{array}$ & $\begin{array}{c}\text { Non- } \\
\text { adherent }\end{array}$ & $\begin{array}{c}\text { Non- } \\
\text { adherent }\end{array}$ & $\begin{array}{c}\text { Non- } \\
\text { adherent }\end{array}$ & $\begin{array}{c}\text { Non- } \\
\text { adherent }\end{array}$ \\
\hline 0.0625 & 770 & $\begin{array}{c}\text { Non } \\
\text { adherent }\end{array}$ & $\begin{array}{c}\text { Non- } \\
\text { adherent }\end{array}$ & $\begin{array}{c}\text { Non- } \\
\text { adherent }\end{array}$ & $\begin{array}{c}\text { Non- } \\
\text { adherent }\end{array}$ & $\begin{array}{c}\text { Non- } \\
\text { adherent }\end{array}$ \\
\hline 3125 & 0,1180 & $\begin{array}{c}\text { Non } \\
\text { adherent }\end{array}$ & $\begin{array}{c}\text { Non- } \\
\text { adherent }\end{array}$ & $\begin{array}{c}\text { Non- } \\
\text { adherent }\end{array}$ & $\begin{array}{c}\text { Non- } \\
\text { adherent }\end{array}$ & $\begin{array}{c}\text { Non- } \\
\text { adherent }\end{array}$ \\
\hline 0.015625 & 0,1015 & $\begin{array}{c}\text { Non } \\
\text { adherent }\end{array}$ & $\begin{array}{c}\text { Non- } \\
\text { adherent }\end{array}$ & $\begin{array}{c}\text { Non- } \\
\text { adherent }\end{array}$ & $\begin{array}{c}\text { Non- } \\
\text { adherent }\end{array}$ & $\begin{array}{c}\text { Non- } \\
\text { adherent }\end{array}$ \\
\hline 0.007813 & 0,2343 & $\begin{array}{c}\text { Moderat } \\
\mathrm{e}\end{array}$ & $\begin{array}{c}\text { Non- } \\
\text { adherent }\end{array}$ & $\begin{array}{c}\text { Non- } \\
\text { adherent }\end{array}$ & $\begin{array}{c}\text { Non- } \\
\text { adherent }\end{array}$ & $\begin{array}{c}\text { Non- } \\
\text { adherent }\end{array}$ \\
\hline 0.003906 & 0,2028 & $\begin{array}{c}\text { Moderat } \\
\mathrm{e}\end{array}$ & $\begin{array}{c}\text { Non- } \\
\text { adherent }\end{array}$ & $\begin{array}{c}\text { Non- } \\
\text { adherent }\end{array}$ & $\begin{array}{c}\text { Non- } \\
\text { adherent }\end{array}$ & Weak \\
\hline 0.001953 & 0,4350 & Strong & $\begin{array}{c}\text { Non- } \\
\text { adherent }\end{array}$ & $\begin{array}{c}\text { Non- } \\
\text { adherent }\end{array}$ & Weak & Medium \\
\hline 0.000977 & $\begin{array}{c}\text { Non- } \\
\text { adherent }\end{array}$ & $\begin{array}{c}\text { Non- } \\
\text { adherent }\end{array}$ & $\begin{array}{c}\text { Non- } \\
\text { adherent }\end{array}$ & $\begin{array}{c}\text { Non- } \\
\text { adherent }\end{array}$ & Weak & \begin{tabular}{|c|} 
Non- \\
adherent
\end{tabular} \\
\hline
\end{tabular}

\section{DISCUSSION}

Major issues posed by formation of biofilm is the adaptation of bacteria in the environment they are found. This means that once bacteria aren't in their natural planktonic form they develop new mechanisms which aren't naturally found in the given bacterial species [13]. The mechanisms are normally presented as resistance to certain biocides and antibiotics, also the ability of bacterial return and recruitment of additional planktonic bacteria is noted [14]. Identification of signaling molecules is of utmost importance due to them being the initiation step in biofilm formation [15]. Novel methods are required in order to deal with the biofilms whether it is preventing their formation by affecting the planktonic microbes or eradication of mature biofilm alongside its residents [15].

Silver throughout history was used as a primitive means of disinfection, starting from using silver coating in goblets and tableware hence the name silverware which was believed to prevent disease [16]. With advancements in science and technology silver maintained its place as a metal which is precious as gold in its own unique ways [17].

Main aim of the this research was to determine if the silver nitrate and silver nanoparticles aside from effects as biocides, affect the biofilm formation clinical strains of $\mathrm{S}$. aureus (MSSA and MRSA) who are not biofilm formers and in S. aureus (ATCC 25923) which had medium biofilm forming capacity. The results of the study indicate that the biofilm formation was quenched in the case of S. aureus (ATCC 25923) using silver nitrate and at the concentration of 0.625 $\mathrm{mM}$ it was reduced from medium to non-adherent, while at concentration of $0.3125 \mathrm{mM}, 0.15625 \mathrm{mM}, 0.0781 \mathrm{mM}, 0.039$ $\mathrm{mM}$ and $0.0195 \mathrm{mM}$ and it was reduced from medium to weak, biofilm returned its intensity back to medium at concentration of $0.00975 \mathrm{mM}$. For case of S. aureus (MSSA) biofilm was increased from non-adherent to weak at concentrations $0.3125 \mathrm{mM}$ and $0.00061 \mathrm{Mm}$ and 0.00030 $\mathrm{mM}$. MRSA did not form biofilm at any of the given concentrations. For the nanoparticles synthesized with green synthesis method S. aureus (ATCC 25923) at concentrations 1 $\mathrm{mM}, 0.5 \mathrm{mM}$ and $0.25 \mathrm{mM}$ the intensity of biofilm has changed from medium to non-adherent, the intensity returned at concentration $0.125 \mathrm{mM}$ and has maintained its intensity to the lowest concentration of $0.0019531 \mathrm{mM}$. S. aurues (MSSA and MRSA) did not form biofilm at any of the concentrations tested. In case of silver nanoparticles S. aureus (ATCC 25923) from medium biofilm has changed at concentration of 0.00098 $\mathrm{mM}$ where weak biofilm has formed, in higher concentrations no biofilm has formed. Both S. aureus MSSA and MRSA did not form biofilm in any of the concentrations.

Second observation was made after 48 hour incubation period, where following results were obtained. Staphylococcus aureus ATCC when exposed to silver nitrate has changed its biofilm intensity from non-adherent to weak at concentrations $0.6 \mathrm{mM}, 0.0195 \mathrm{mM}$ and $0.00975 \mathrm{mM}$ while at other concentrations it remained non-adherent. S. aureus (MSSA) and S. aureus (MRSA) did not show any changes in their biofilm intensity from non-adherent. When tested with silver nanoparticles synthesized with Aloe Vera S. aureus (ATCC 25923) change is noticed at concentrations $0.25 \mathrm{mM}$ and $0.15625 \mathrm{mM}$ when compared to other concentrations where no biofilm has formed. S. aureus MSSA has changed its intensity from non-adherent to weak at concentration 0.125 $\mathrm{mM}$ and MRSA has also changed its intensity at $0.5 \mathrm{mM}$ to weak biofilm from non-adherent.

The obtained results were similar compared to the results of other studies however the only difference was in the used methodology which in the study of (Ghotaslou et al Bahari et al Aliloo et al Eshlaghi) was the test tube method. [19] [20]. While the major focus of this research was effect of silver nitrate and silver nanoparticles on the biofilm formation, some other discoveries were made. The duration the medium exposed to the environment inside an incubator set at 37 degrees Celsius is maximum two and half days, everything beyond that point is subjected to evaporation and condensation which can lead towards contamination and other issues related to concentration and longevity of the experiment. Limitation of the study was the number of tested S. aureus strains where in future experiments this number should be increased to get statistically more significant results. Improvements could be made in overall design and storage of 96 well plates with specialized parafilm tape which would prevent evaporation of medium providing longer longevity of samples in the wells. Follow-up on the current study can be performed with different species of bacteria which are notable in hospital environments or closely related to the medical conditions. More detailed and precise methods of synthesis can also be taken in consideration alongside more quality of life improvements when it comes to potential reductions in contamination and agglomeration of nanoparticles. Also possible tests on live tissue cultures can be also performed to 
observe the same concentrations of silver nanoparticles and how they affect the human cell lines. Major contribution of this study was the determination of concentrations which may hinder or affect the bacteria by not serving as signaling molecule to form biofilm. This research can be used in future development and improvements in methods which can serve as models for various treatments. Those treatments can be novel methods in dealing with biofilms which may cause serious issues in healthcare and recovery. Additional strains must be tested to obtain their specific sensitivity to the given conditions and to obtain broader picture.

\section{REFERENCES}

[1] Sutherland, I. (2001). Biofilm exopolysaccharides: a strong and sticky framework. Microbiology, 147(1), 3-9. doi: 10.1099/00221287-147-1-3

[2] Allison, D., \& Sutherland, I. (1987). The Role of Exopolysaccharides in Adhesion of Freshwater Bacteria. Microbiology, 133(5), 1319-1327. doi: 10.1099/00221287-133-5-1319

[3] Christensen, B. (1989). The role of extracellular polysaccharides in biofilms. Journal Of Biotechnology, 10(3-4), 181-202. doi: 10.1016/0168-1656(89)90064-3

[4] James, G., Beaudette, L., \& Costerton, J. (1995). Interspecies bacterial interactions in biofilms. Journal Of Industrial Microbiology, 15(4), 257-262. doi: 10.1007/bf01569978

[5] Gugala, N., Lemire, J. and Turner, R. (2017). The efficacy of differen anti-microbial metals at preventing the formation of, and eradicating bacterial biofilms of pathogenic indicator strains. The Journal of Antibiotics, 70(6), pp.775-780.

[6] Alexander, J. (2009). History of the Medical Use of Silver. Surgical Infections, 10(3), 289-292. doi: 10.1089/sur.2008.9941

[7] Jung, W., Koo, H., Kim, K., Shin, S., Kim, S. and Park, Y. (2008). Antibacterial Activity and Mechanism of Action of the Silver Ion in Staphylococcus aureus and Escherichia coli. Applied and Environmental Microbiology, 74(7), pp.2171-2178.

[8] Goswami, S., Sahareen, T., Singh, M., \& Kumar, S. (2015). Role of biogenic silver nanoparticles in disruption of cell-cell adhesion in Staphylococcus aureus and Escherichia coli biofilm. Journal Of
Industrial And Engineering Chemistry, 26, 73-80. doi 10.1016/j.jiec.2014.11.017

[9] Jung, W., Koo, H., Kim, K., Shin, S., Kim, S. and Park, Y. (2008). Antibacterial Activity and Mechanism of Action of the Silver Ion in Staphylococcus aureus and Escherichia coli. Applied and Environmental Microbiology, 74(7), pp.2171-2178.

[10] Maaz, K. (n.d.). Silver nanoparticles.

[11] Chou, K. and Ren, C. (2000). Synthesis of nanosized silver particles by chemical reduction method. Materials Chemistry and Physics, 64(3), pp.241-246.

[12] Swain, A. (2016). Review on Green Synthesis of Silver Nanoparticles by Physical, Chemical and Biological Methods. International Journal of Scientific \& Engineering Research, 7(10), pp.551-554

[13] Skillman, L., Sutherland, I., \& Jones, M. (1998). The role of exopolysaccharides in dual species biofilm development. Journal Of Applied Microbiology, 85(S1), 13S-18S. doi: 10.1111/j.13652672.1998.tb05278.x

[14] Presence of icaA and icaD genes and slime production in a collection of staphylococcal strains from catheter-associated infections

[15] Mayer, C., Moritz, R., Kirschner, C., Borchard, W., Maibaum, R. Wingender, J., \& Flemming, H. (1999). The role of intermolecular interactions: studies on model systems for bacterial biofilms. International Journal Of Biological Macromolecules, 26(1), 3-16. doi 10.1016/s0141-8130(99)00057-4

[16] James, G., Beaudette, L., \& Costerton, J. (1995). Interspecies bacterial interactions in biofilms. Journal Of Industrial Microbiology, 15(4), 257-262. doi: 10.1007/bf01569978

[17] Alexander, J. (2009). History of the Medical Use of Silver. Surgical Infections, 10(3), pp.289-292.

[18] Kędziora, A., Speruda, M., Krzyżewska, E., Rybka, J., Łukowiak, A and Bugla-Płoskońska, G. (2018). Similarities and Differences between Silver Ions and Silver in Nanoforms as Antibacterial Agents. International Journal of Molecular Sciences, 19(2), p.444.

[19] Silver nanoparticles for the inhibition of Staphylococcus aureus (2015). Entreciencias: Diálogos en la Sociedad del Conocimiento, 3(7), pp.133-142.

[20] Ghotaslou, R., Bahari, Z., Aliloo, A., Gholizadeh, P. and Eshlaghi, B. (2017). THE IN VITRO EFFECTS OF SILVER NANOPARTICLES ON BACTERIAL BIOFILMS. Journal of Microbiology, Biotechnology and Food Sciences, 6(4), pp.1077-1080 